\title{
Reporting of acute pancreatitis by radiologists-time for a systematic change with structured reporting template
}

\author{
Aman Khurana ${ }^{1} \cdot$ Leslie W Nelson $^{1}$. Charles B Myers ${ }^{1} \cdot$ Fatih Akisik $^{2}$ - Brooke R. Jeffrey ${ }^{3} \cdot$ Frank H. Miller $^{4}$. \\ Pardeep Mittal $^{5} \cdot$ Desiree Morgan ${ }^{6} \cdot$ Koenraad Mortele $^{7} \cdot$ Peter Poullos $^{3} \cdot$ Dushyant Sahani $^{8} \cdot$ Kumar Sandrasegaran $^{9}$. \\ Temel Tirkes $^{2} \cdot$ Atif Zaheer $^{10} \cdot$ Bhavik N. Patel $^{3}$
}

Published online: 18 March 2020

๑) Springer Science+Business Media, LLC, part of Springer Nature 2020

\begin{abstract}
Acute pancreatitis has a wide array of imaging presentations. Various classifications have been used in the past to standardize the terminology and reduce confusing and redundant terms. We aim to review the historical and current classifications of acute pancreatitis and propose a new reporting template which can improve communication between various medical teams by use of appropriate terminology and structured radiology template. The standardized reporting template not only conveys the most important imaging findings in a simplified yet comprehensive way but also allows structured data collection for future research and teaching purposes.
\end{abstract}

Keywords Acute pancreatitis · Revised Atlanta classification - Interstitial edematous $\cdot$ Necrotizing pancreatitis · Peripancreatic fluid collections $\cdot$ Pseudocyst $\cdot$ Radiology reporting template

Aman Khurana

aman.k@uky.edu

1 Division of Abdominal Radiology, Department of Radiology, University of Kentucky, Lexington, KY, USA

2 Department of Radiology, Indiana University, Indianapolis, USA

3 Department of Radiology, Stanford University, Stanford, USA

4 Department of Radiology, Northwestern University, Chicago, USA

5 Department of Radiology, Medical College of Georgia, Augusta, USA

6 Department of Radiology, University of Alabama at Birmingham, Birmingham, USA

7 Department of Radiology, Beth Israel Deaconess Medical Center, Boston, USA

8 Department of Radiology, University of Washington, Seattle, USA

9 Department of Radiology, Mayo Clinic Arizona, Phoenix, USA

10 Department of Radiology, Johns Hopkins University, Baltimore, USA

\section{Background}

Acute pancreatitis (AP) is a common inflammatory process affecting the pancreas with variable disease severity, ranging from a mild course treated with conservative management to severe progressive disease resulting in major morbidity and mortality [1]. The majority of acute pancreatitis episodes are secondary to gallstones (22.7\%) and alcohol use (22.5\%) $[2,3]$. Another relative prevalent etiology is hypertriglyceridemia $(3.7 \%)$, while fourty-eight percent of cases have no known etiology [3]. Iatrogenic injury, infections, neoplasia, structural abnormalities, inflammatory bowel disease, toxins, trauma and drugs are less common etiologies of acute pancreatitis [4].

Per the Revised Atlanta classification, the diagnosis of acute pancreatitis is defined by at least 2 out of the 3 following features: epigastric pain often radiating to the back, biochemical findings of serum amylase and lipase at least three times the normal limit or radiologic imaging features suggestive of acute pancreatitis [5]. The Revised Atlanta classification also differentiates between an early and late phase of acute pancreatitis [6]. The early phase occurs within the first week of disease onset with pathology caused by early inflammation secondary to peripancreatic edema and ischemia. The severity of this early phase is based on 
patient's clinical presentation as the imaging findings sometimes may not correlate well [7]. This is a dynamic phase where the patient will either resolve or continue to progress to a more severe presentation. The late phase begins within the second week of onset and can last weeks to months and is characterized by the presence of local complications and systemic inflammation. The various complications are able to be characterized by radiological imaging while organ failure continues to be a clinical diagnosis [8]. The prevalence of pancreatitis has increased by a total of $18 \%$ from 2006 to 2014. When divided by age groups, the incidence of acute pancreatitis in Emergency Department visits showed an increase of 9.2\% (ages 18-45) and 8.6\% (ages 45-65) from 2006 to 2012. This is in contrast to older patients which demonstrated a decrease in incidence $-13.4 \%$ (ages 65-85) and $-20.1 \%$ (greater than 85 years old) [9]. The obesity pandemic appears to have increased the incidence and severity of acute pancreatitis secondary to the risk of gallstones, diabetes mellitus, hypertriglyceridemia, incretin based medication and endoscopic interventions for management [10]. In 2014, there were approximately 351,526 annual Emergency Department visits for the diagnosis of acute pancreatitis and 39,413 visits for chronic pancreatitis. Of these visits, $70 \%$ of acute and $25.3 \%$ of chronic pancreatitis were admitted to the hospital with a median length of stay of 3 days. There were $1571(0.5 \%)$ and $13(0.03 \%)$ hospital deaths related to acute and chronic pancreatitis, respectively. The aggregate charges for the pancreatitis treatment in 2014 was $\$ 10,486,824,627$ and aggregate costs were $\$ 2,772,024,840$ [11].

\section{Revisiting the 1992 Atlanta Classification}

The 1992 Atlanta Classification System for Acute Pancreatitis was the first standardized system proposed to create a standardized communication for gastroenterologists, pathologists, radiologists and surgeons with common terms of acute pancreatitis and associated complications [7] (Table 1). At the time of its creation, the classification made great strides by attempting to standardize the diagnosis of acute pancreatitis and provide a framework to consistently and reliable define its severity and categorize its complications.

As a greater understanding of the pathogenesis and natural evolution of the disease occurred, shortcomings of the original Atlanta classification schema were uncovered. As an example, one major limitation was its lack of specific radiologic criteria for defining complications associated with AP, especially when describing pancreatic and peripancreatic fluid collections. The 1992 classification defined the following four complications: acute fluid collection, acute pseudocyst, pancreatic abscess, and pancreatic necrosis (Table 1) [12]. These definitions were vague and failed to adequately describe isolated peripancreatic collections as well as collections that contained both solid and fluid components. Ultimately, this contributed to the widespread misuse and incontinency in applying the terms. This limitation was highlighted in a study that demonstrated poor interobserver agreement between radiologists using the original classification for characterizing peripancreatic collections on CT [13].

The original classification did not differentiate pancreatic and peripancreatic necrosis nor did it delineate sterile vs infected necrosis. Properly describing and differentiating these entities is crucial because there is a profound impact on prognosis and management. In 2012 the Revised Atlanta Classification sought to resolve these limitations by updating terminology, types of AP, definitions of complications, and separating the disease temporally into two distinct phases. Previously, there have been several terms within the nomenclature for describing complications of acute pancreatitis that have been ambiguous and/or improperly used leading to much clinical confusion. One such example was the term "pancreatic abscess" that had been defined as "a circumscribed intra-abdominal collection of pus, usually in proximity to the pancreas, containing little or no pancreatic necrosis, which arises as a consequence of acute pancreatitis or pancreatic trauma" [12]. Infected necrotic collections are far more common than true pancreatic abscesses and the distinction between the two entities is important because of a profound impact on morbidity and mortality as well as management [14]. In order to truly differentiate the two entities, positive fluid cultures were required in the absence of necrosis to accurately define a collection as a pancreatic abscess. This was impractical and only contributed to delayed or inappropriate care. Ultimately, due to its ubiquitous misuse the term pancreatic abscess was removed from the nomenclature in the revised Atlanta classification in 2012 and has been replaced by infected or sterile necrosis as described below.

\section{The Revised 2012 Atlanta Classification}

The Revised Atlanta Classification (RAC) of AP published in 2012 to address the growing limitations of the original AC schema (Table. 1). Greater understanding of the pathophysiology and natural evolution of the disease resulted in defining two distinct phases of AP: early ( $<1$ week after onset) and late ( $>1$ week after onset). Severity and management during the early phase are solely based on clinical parameters, whereas in the late phase clinical and radiographic findings influence management and severity. An important change in the RAC was the development of a three-tiered system for grading AP severity as mild, moderately severe, or severe based on the presence and duration of organ 
Table 1 Differences between types of acute pancreatitis (a) and fluid collections (b) in the 1992 and 2012 Atlanta Classifications for Acute Pancreatitis

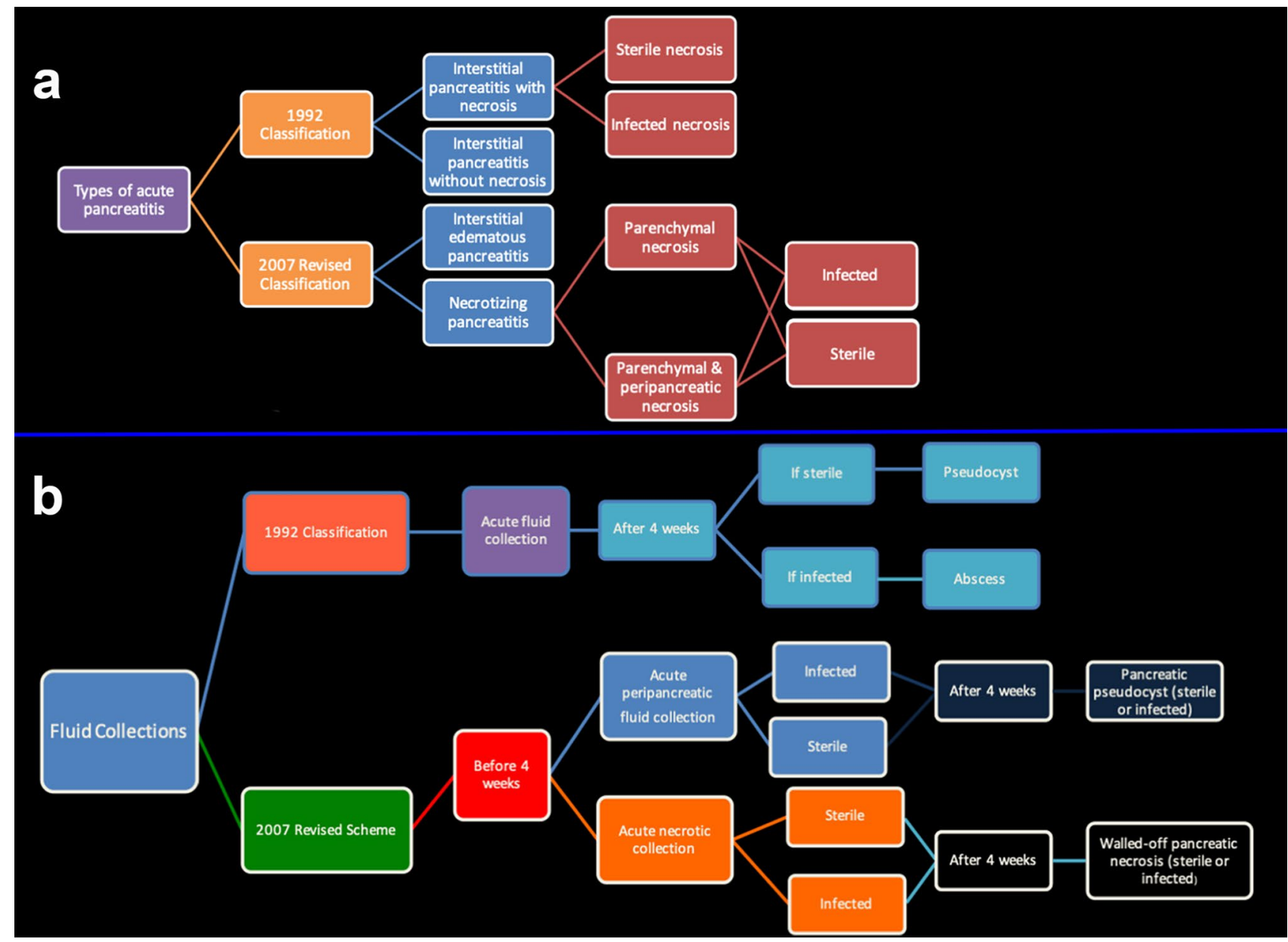

Please note that it takes approximately 4 weeks to form a well-defined capsule and therefore the revised classification emphasizes on this point. Some collections may form capsules before or after this time interval, in those cases the imaging characteristics are given preference over this time interval

failure. Mild pancreatitis is defined by lack of organ failure, lack of local or systemic complications and self-resolving course [15]. Moderately severe acute pancreatitis defined by transient organ failure $(<48 \mathrm{~h})$ or local or systemic complications with absence of persistent organ failure. Moderately severe acute pancreatitis may require treatment and does have slightly increased mortality (approximately 2\%) [8]. Severe AP is characterized by organ failure greater than 48 $\mathrm{h}$, often accompanied by local and systemic complications or death. Approximately $15-20 \%$ of acute pancreatitis will advance to severe acute pancreatitis [16]. The duration of organ failure is a marker of poor outcome and associated with higher morality, therefore requires more aggressive management [17].

To facilitate prompt and appropriate management in patients with AP, the RAC eliminated and outdated ambiguous terms such as infected pseudocyst, phlegmon, hemorrhagic pancreatitis and persistent acute pancreatitis, that lead to confusion and often delayed or inappropriate treatment. Perhaps the greatest improvement of the RAC was its development of specific criteria for categorizing complications of AP. This was a significant limitation of the original classification that contributed to poor interobserver agreement in defining pancreatic and peripancreatic fluid collections. The RAC distinguishes complications as either acute ( $<4$ weeks after onset) or delayed ( $>4$ weeks after onset) and further subcategorizes based on necrotic vs interstitial types of pancreatitis [8] (Table 1). It should be noted that it takes approximately 4 weeks to form a well-defined capsule and therefore the revised classification emphasizes on this point. Some collections may form capsules before or after this time interval, in those cases the imaging characteristics are given preference over this time interval. Acute complications are categorized as either acute peripancreatic fluid collection (APFC) or acute necrotic collection (ANC) and delayed complications are defined as either pancreatic 
pseudocyst (PC) or walled-off necrosis (WON). Any of these collections can develop a superimposed infection, therefore they are referred to as either sterile or infected. Each type of collection is managed uniquely, and thus, it was essential to standardize definitions so that complications can be accurately and consistently reported to surgeons, endoscopists, interventionalists, and clinicians with greater clarity.

\section{The Role of Imaging}

Imaging is typically not indicated in the early course of AP because the diagnosis can be made on clinical and biochemical data and early imaging $(<72 \mathrm{~h})$ can often be misleading because of underestimation of the true degree of parenchymal involvement as well as the inability to reliably assess complications in the early course of the disease [7]. Furthermore, early imaging has not been found to improve clinical outcomes and in some studies, it has been suggested that it may prolong hospitalization [18]. IAP/APA guidelines state that initial imaging is indicated in the following circumstances: (1) Diagnosis is unclear (2) Confirm the clinical prediction of severe pancreatitis (3) Clinical deterioration or failure to respond to conservative measures [19]. Imaging still plays a monumental role in the characterization and management of complications of AP. Contrast-enhanced CT is the modality of choice in the initial assessment of patients with AP to determine the etiology, define the severity and identify complications and early signs of necrosis [2]. Defined by the current Revised Atlanta classification there are currently two main morphologic subtypes of AP based on radiographic findings: interstitial edematous pancreatitis (IEP; $85 \%$ of cases) and necrotizing pancreatitis (NP; $15 \%$ of cases) [8]. The characteristic radiographic findings of IEP include: focal or diffuse pancreatic edema, diffuse pancreatic enhancement, peripancreatic fat stranding and/or haziness (Fig. 1). NP demonstrates a relatively more diverse array of morphologies dependent on the location of necrosis. The location of necrosis differentiates the three patterns of NP: pancreatic (5\%), peripancreatic $(20 \%)$ or combined (75\%) (Fig. 2). In pancreatic NP, the key radiographic finding is focal or diffuse non-enhancement of the pancreatic parenchyma without the presence of a peripancreatic necrotic collection (Fig. 3). Peripancreatic NP usually demonstrates diffuse pancreatic enhancement, but will have mixed solid/fluid necrotic collection(s) in the peripancreatic tissues (Figs. 2, 4). Combined NP will show diffuse or focal areas of pancreatic non-enhancement in addition to adjacent necrotic peripancreatic solid/fluid collections (Figs. 2, 4) [7]. Imaging within the first $24 \mathrm{~h}$ of symptom onset may fail to demonstrate necrosis as interstitial edema and early necrosis can look identical (heterogeneous enhancement). If present, the region(s) of necrosis will evolve over time and develop a characteristic non-enhancement pattern which is best evaluated by contrast enhanced CT. For these reasons, $72 \mathrm{~h}$ after the onset of symptoms is considered the ideal time for imaging patients in order to confidently identify acute complications of AP [2]. In a clinical setting, determining necrotic versus interstitial edematous pancreatitis is critically important as it has a profound impact on prognosis.
Fig. 1 Axial CECT image of diffuse interstitial edematous pancreatitis and the proposed template demonstrating homogeneous enhancement and diffuse enlargement of the pancreas with surrounding inflammation (arrow)

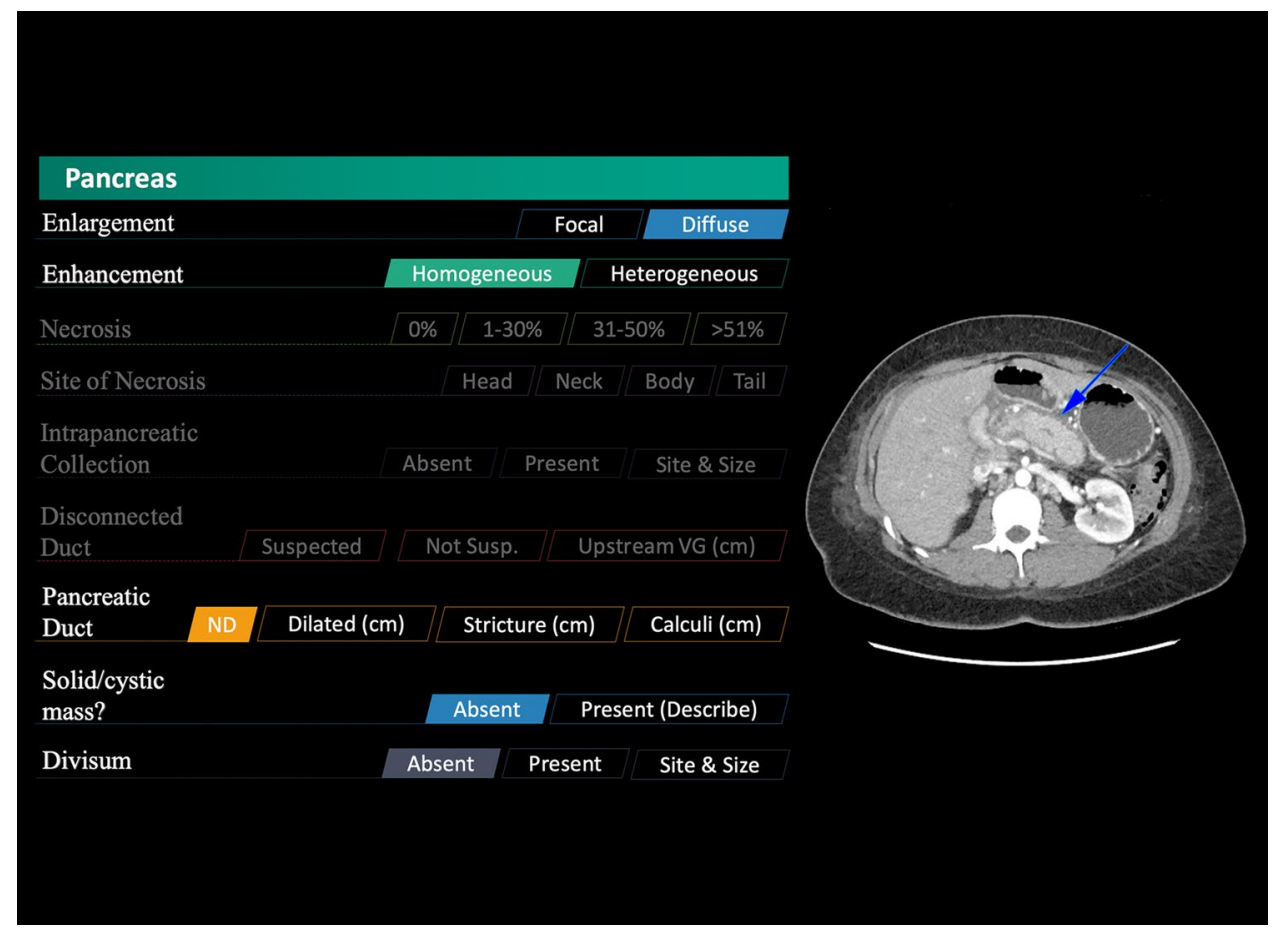



pancreatic parenchymal necrosis alone with heterogeneous nonenhancement of the pancreas (arrow) b peripancreatic necrosis alone with heterogeneous area of non-enhancement with non-liquified components in the peripancreatic fat (arrow) but with normally enhancing pancreas parenchyma (arrowhead) and $\mathbf{c}$ pancreatic and peripancreatic necrosis with peripancreatic necrotic collection (arrow) and heterogeneous non-enhancement of the pancreas indicating necrosis (arrowhead)
Fig. 2 Axial CECT images of $\mathbf{a}$

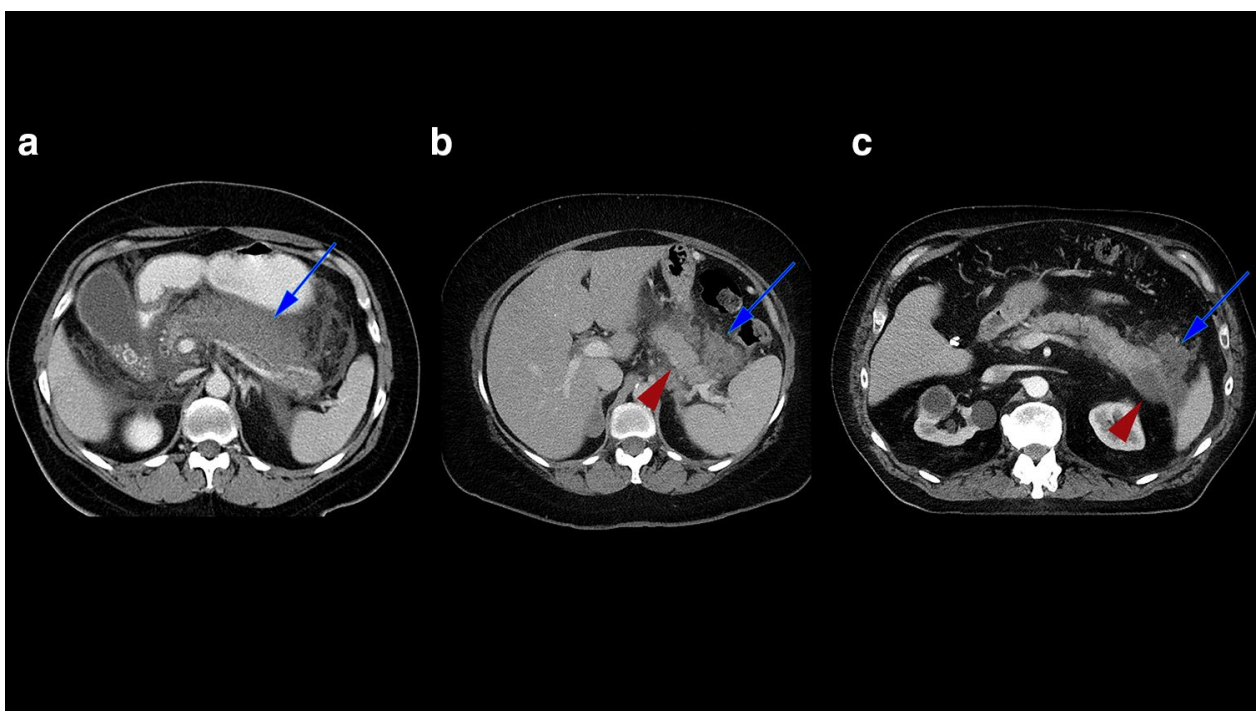

Fig. 3 Axial CECT image of diffuse necrotizing pancreatitis and the proposed template demonstrating non-enhancing pancreatic parenchyma (demarcated by arrows) and surrounding inflammatory changes

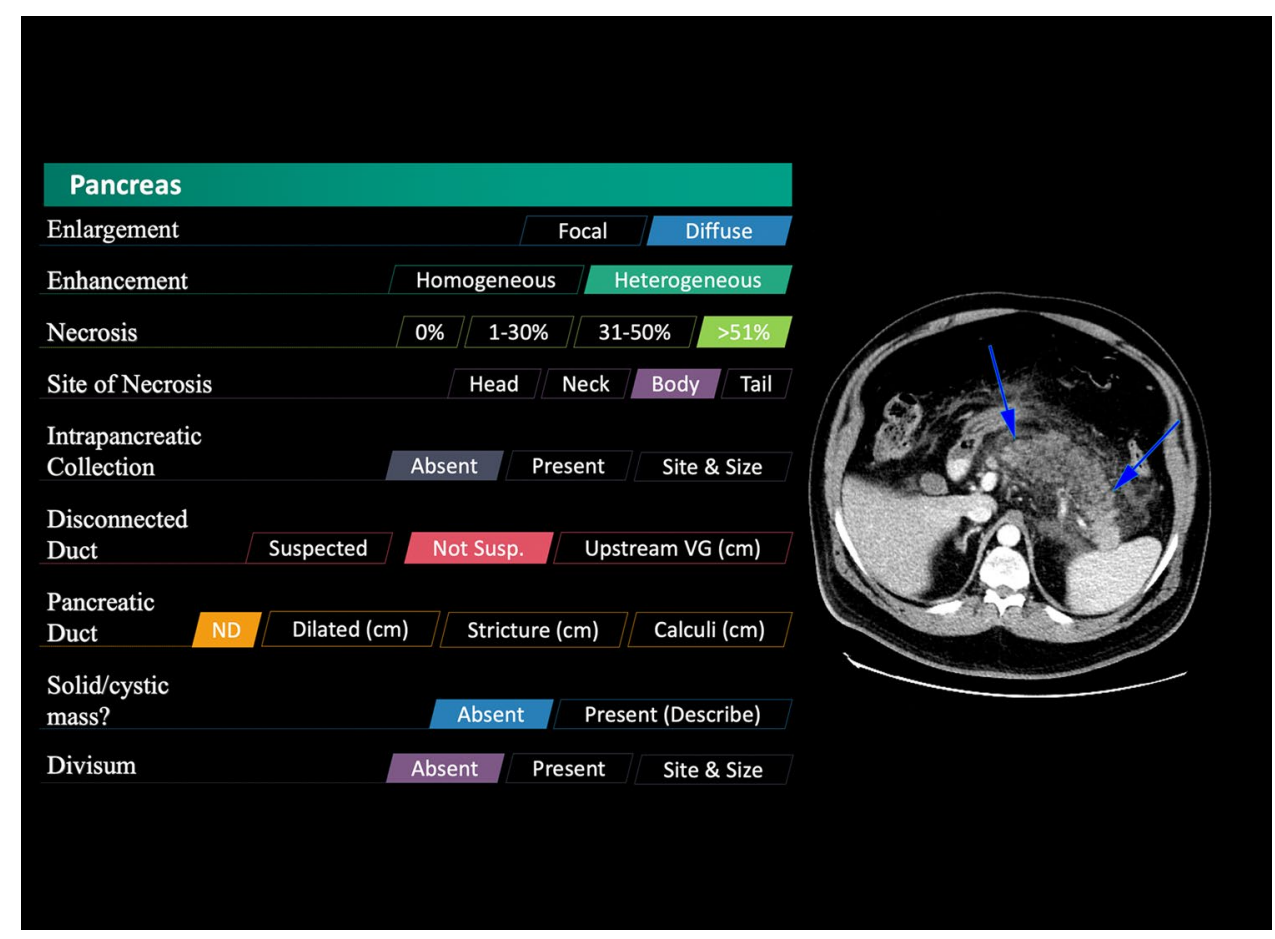

Patients diagnosed with IEP have a mortality rate of approximately $3 \%$ compared to patients with NP, which have a mortality rate of $17 \%$ and up to $30 \%$ if superimposed infection is present [20].

Abdominal CT has contributed significantly to the assessment of acute pancreatitis by determining the degree of severity, extent of necrosis, fluid collections, pseudocysts, abscess and prognosis [21]. According to the 2013 Working Group of the International Association of Pancreatology (IAP) and American Pancreatic Association (APA) IAP/APA acute pancreatitis guidelines when a patient presents with diagnostic uncertainty of acute pancreatitis there is a strong recommendation to complete initial CT assessment [19]. However, optimal timing of initial CT imaging is at least 72-96 $\mathrm{h}$ after the onset of symptom presentation. Performing a CT scan earlier than this has been shown to have low yield and no clinical management implantations [22]. Further indications for CT imaging include confirmation of severity based on clinical course, failure to respond to conservative treatment and clinical deterioration. Follow up imaging with CT is indicated when there is a lack of clinical improvement, especially when there is consideration for an invasive intervention [19]. MR with fluid sensitive sequences helps better 
Fig. 4 a Proposed template and axial CECT showing peripancreatic inflammation, areas of pancreatic non-enhancement, and surrounding non-encapsulated fluid (arrow) classified as acute necrotic collection and b Proposed template and axial CECT showing peri-pancreatic inflammation, areas on pancreatic non-enhancement and surrounding non-encapsulated acute necrotic collection with gas (arrow)

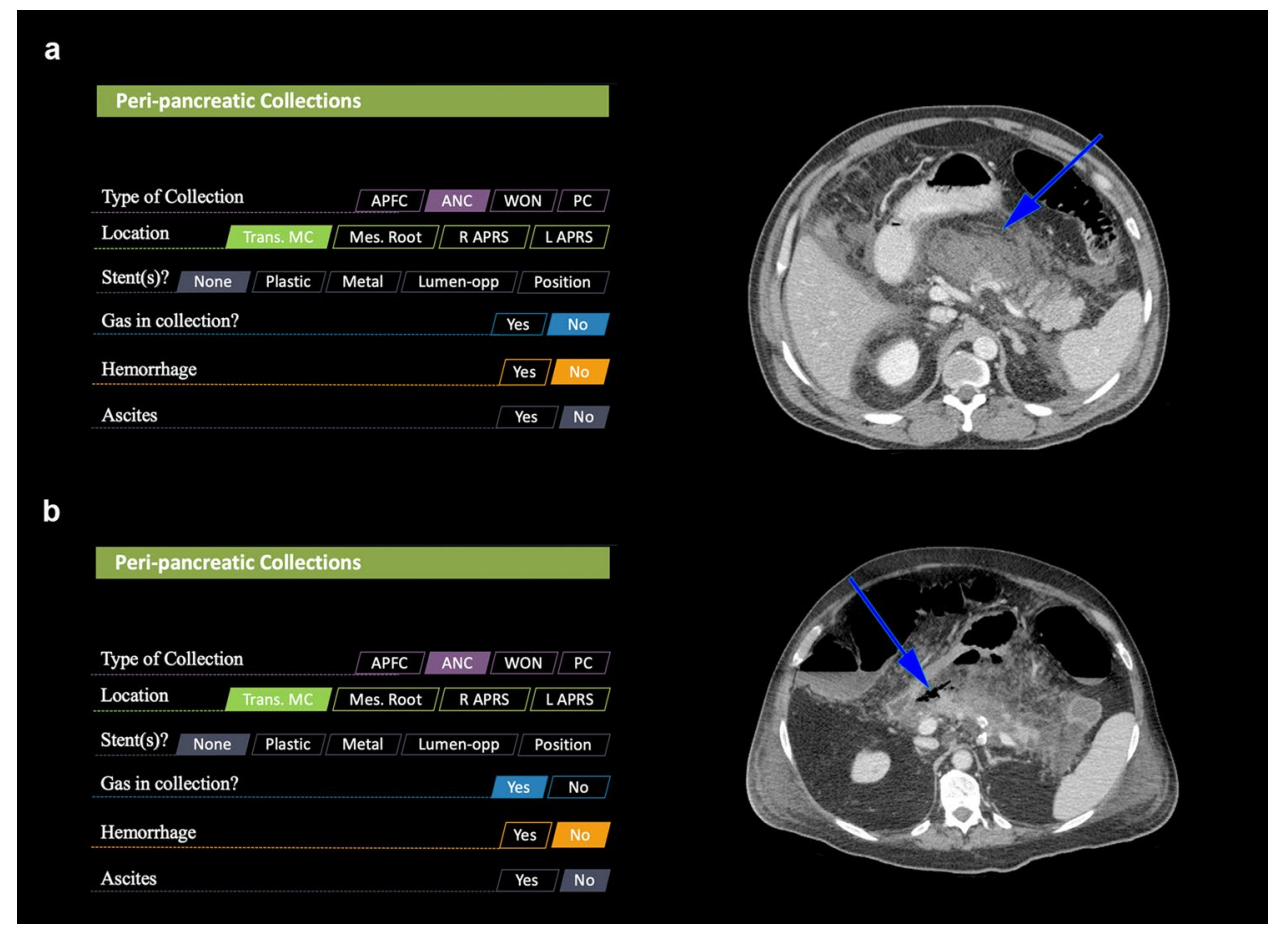

assess the presence of debris within a fluid collection and the presence of ductal disruption [23].

\section{Management}

The management of acute pancreatitis is mostly supportive care, however, the challenge of treatment occurs when the patient presents with severe disease and complications [24]. Therefore a multidisciplinary approach is utilized when there is an indication for intervention. Determining the amount of necrosis (less than $30 \%, 30-50 \%$ or greater than 50\%) and the location of necrosis (head, body or tail) assists in the determination of the patient's potential need for operative intervention [25]. A patient with presence of pancreatic necrosis is ten times more likely to have surgical intervention [26]. Another example of the role of imaging in altering the management is the diagnosis of infected necrosis, which is made when the patient experiences fever, develops increasing inflammatory markers and demonstrates gas in the peripancreatic collection on imaging, at which point percutaneous fine needle aspiration of the collection is not required to confirm the diagnosis as the clinical and radiological findings are sufficient for a diagnosis [19]. Radiological modalities not only assist with the patient's diagnosis, but can also help reveal the cause of acute pancreatitis. Visualization of the gallbladder and biliary tract can further evaluate pathology as biliary or non-biliary etiology, as gallstones are the most common cause of acute pancreatitis accounting for at least $23-40 \%$ of cases $[16,27]$.
Management of AP is largely influenced by two main factors: severity and complications. One of the most crucial steps in initial management of a patient presenting with AP is determining the etiology of pancreatitis so that any reversible cause can be addressed promptly. Once the etiology has been addressed, the severity of the disease needs to be determined in order to appropriately triage patients to ensure that the critically ill receive the appropriate level of care. The IAP/APA evidence-based guidelines for the management of acute pancreatitis state that the best predictor of AP severity/prognosis at the time of admission and at $48 \mathrm{hr}$ is the presence of Systemic Inflammatory Response Syndrome (SIRS) [19]. Any patient that is classified as severe should be admitted or transferred to the ICU for close surveillance as mortality in this population has been reported to be as high as $15-85 \%$ [28]. Patients classified with mild disease often have a self-limited course that requires supportive care only. Patients classified with moderately severe or severe disease will require more aggressive care and possibly open or minimally-invasive interventions. Patients with morphologic IEP on imaging often have a mild and self-limited course that typically only requires supportive care. If present, concomitant APFCs often will either regress or mature to pseudocysts, of which the majority of will resolve without requiring intervention. Approximately $25 \%$ of patients with pseudocysts develop symptoms or superimposed infection that in turn requires intervention [29]. Percutaneous or endoscopic catheter drainage are the treatment modalities of choice in the management of infected or symptomatic pseudocysts. 
Patients with morphologic NP on imaging have increased morbidity and mortality requiring intervention far more often than AP patients without concomitant necrosis. Surgeons, endoscopists, interventionalists and radiologists must be able to work together and communicate openly and effectively so that the best treatment plan can be tailored to each individual case of infected NP. At the center of this multidisciplinary approach is the role of the radiologist in communicating the appropriate information that is needed in order to tailor management. Therefore a more comprehensive yet structured reporting template is imperative for clear and concise communication of the most relevant information to the members of the multidisciplinary team.

\section{Current Limitations and Need for Reporting Template}

While the Revised Atlanta classification system has made improvements in the diagnosis and classification of acute pancreatitis, there are still areas for further improvement. For example in one prospective study by Talukdar et al., patients who were originally classified into moderately severe acute pancreatitis with primary infected necrosis had outcomes similar to severe acute pancreatitis, therefore the former disease course should be treated more aggressively [30]. Also, with the current classification, there remains variability among subspecialty and general radiologists as shown by Sternby et al. who demonstrated only fair agreement between the local radiologists and the central expert in diagnosis of non-homogeneous collections and extra-pancreatic necrosis resulting in inconsistent reporting [31]. Further establishment of widespread terminology use and simple identification of complications could continue to improve pancreatitis templates [15]. Until now, however, no radiology reporting templates have been proposed for standardized reporting of acute pancreatitis and its complications.

The RSNA's radiology reporting initiative has been a widely recognized effort to improve reporting practices by creating and managing a library of clear and consistent report templates [32]. Greater standardization could result in more comprehensive reports, better communication and fewer misdiagnoses [33]. Consistent radiologic reports are of paramount importance in assessment of the degree of disease severity and response to therapy in both clinical practice and clinical trials. A prior study compared content, clarity and clinical usefulness of conventional (free text) and structured radiology of body CT scans and found that mean content and clarity satisfaction ratings were significantly higher for standardized reporting when compared to conventional reports [34]. Structured reporting has been introduced in other abdominal imaging pathologies, particularly pancreatic cancer where newer reporting styles have shown superior evaluation of pancreatic cancer and resultant improvement in surgical planning, with increased confidence of surgeons regarding decisions about tumor resectability when structured reports were utilized [35]. Safety checklists are frequently used in surgery as a quality assurance tool to effectively reduce complication rates and mortality in adults undergoing noncardiac surgery [36]. Beyond improved clinical communication, the implementation of checklists in radiology has been demonstrated to reduce variability and error rates [37, 38]. Another key role of structured reporting is its role in facilitating learning among trainees by providing them with a standardized and systematic approach to recognize the key features needed in radiologic reports of patients with specific diseases. Even though implementation of department-wide standardized structured reporting can be problematic, prior work has shown excellent adoption rate (approaching $100 \%$ ) by focusing on automatic population of examination specific reports and more efficient report monitoring for quality assurance and research [39]. Overall, structured reporting aims to provide the benefits of standardization such as clearer communication, comprehensive details and increased accessibility of data for research without compromising radiologists' ability to communicate qualitative findings and opinions.

\section{Proposed Reporting Template}

The proposed reporting template is for acute pancreatitis and is meant to be used with contrast enhanced CT imaging to ensure a complete evaluation of the pancreatic parenchyma and associated vasculature. The template was constructed from a group of expert radiologists who are members of Society of Abdominal Radiology, Pancreatitis Disease Focussed Panel over multiple sessions. The entire reporting template is summarized in Appendix Table 2.

The primary goal of standardized reporting is to ensure proper communication between all sub-specialists involved in the care of the patient. This template incorporates revised Atlanta classification scheme and terminology while maintaining flexibility to add free text for the qualitative aspects of the report. The four broad categories of this template are 1. Pancreas, 2. Peripancreatic collections, 3. Upper abdominal vasculature and 4 . Other. These categories allow comprehensive reporting of pancreatic parenchymal enlargement and enhancement, patency of the pancreatic duct, location and type of peripancreatic collection, presence of gas/infection within and around the pancreas and assessment of peripancreatic vasculature for thrombosis, aneurysm etc.

The first and the most important category of this template is centered around findings related to the pancreas itself. Pancreatic enlargement is classified as focal or diffuse and its enhancement is classified as homogenous or heterogenous 
(Figs. 1, 3). This is most helpful in describing interstitial edematous pancreatitis. Pancreatic necrosis is subclassified by the anatomical region of the organ and percentage of the non-enhancing pancreatic parenchyma, for example $0 \%,<30 \%, 31-50 \%$ and $>51 \%$ subcategories (Fig. 3). These subcategories are clinically important because amount of gland necrosis is predictive of development of superimposed infection, organ failure, and morbidity and mortality, and need for necrosectomy [40]. Evaluation of the pancreatic duct is often not reported in free text reports but in this template we focus on the contiguity of the pancreatic duct in efforts to identify and correctly diagnose disconnected duct syndrome (Fig. 5). In these patients, the disease can be centered about the ductal epithelium and persistent inflammatory collections and fistulae are usually seen. The diagnosis of disconnected duct syndrome is suggested when following findings are encountered, $>2 \mathrm{~cm}$ area of necrosis, viable upstream tissue and extravasation of contrast on ERCP [41]. DPDS can be suggested on cross-sectional imaging, CECT or MRCP, however, pancreatography remains the gold standard for confirmation and ductal characterization [42]. Secretin-enhanced MRCP is an emerging technique that utilizes the physiologic properties of secretin to induce pancreatic exocrine function, which in turn produces optimal ductal morphologic features that can be appreciated on MRCP. The administration of secretin during MRCP results in increased sensitivity for detecting chronic pancreatitis, IPMNs and ductal injuries or variants compared to MRCP without secretin administration [43]. For this reason secretin-enhanced MRCP has been postulated to be a reliable noninvasive alternative for diagnosing DPDS. However, secretin-enhanced MRCP has not yet been proven to have greater sensitivity than ERCP in determining the site of ductal disconnection, therefore it currently assumes a complementary role to traditional endoscopy in diagnosing DPDS [44]. Additional findings related to pancreatic duct include presence of dilation, strictures and calculi. Intrapancreatic collections can be seen with pancreatic parenchymal necrosis and disconnected duct syndrome. Lastly, other ancillary findings related to pancreas are reported such as presence of pancreatic divisum or solid/cystic mass.

The second most important category in the reporting template is Peripancreatic Fluid Collections. They are subcategorized as 1 . acute peripancreatic fluid collections which are associated with interstitial edematous pancreatitis or 2 . acute necrotic collections associated with necrotizing pancreatitis provided if the retroperitoneal findings are visualized less than 4 weeks from symptom onset. Acute peripancreatic fluid collection should be homogenous density and adjacent to the pancreas, intrapancreatic fluid collections and collections with variable attenuation should be considered as necrosis [23]. Acute necrotic collections may reside within the pancreatic parenchyma and may be associated with pancreatic duct disruption (Fig. 5). Similarly, pseudocyst and walled off necrosis categories are used when encapsulated collections associated with interstitial and necrotizing pancreatitis, respectively, are more than 4 weeks old respectively (Figs. 6, 7). All of these collections can be sterile or infected. Unlike pseudocysts, walled off necrosis collections commonly involve pancreatic parenchyma. The location
Fig. 5 a Proposed template with axial CECT image of focal area of intraparenchymal pancreatic necrosis (arrow) which is suspicious for a disconnected pancreatic duct and $\mathbf{b}$ Coronal MRCP confirming disconnected duct syndrome as no connection between the pancreatic ductal segments (arrowheads) is visualized and the intraparenchymal fluid collection is again seen (arrow)

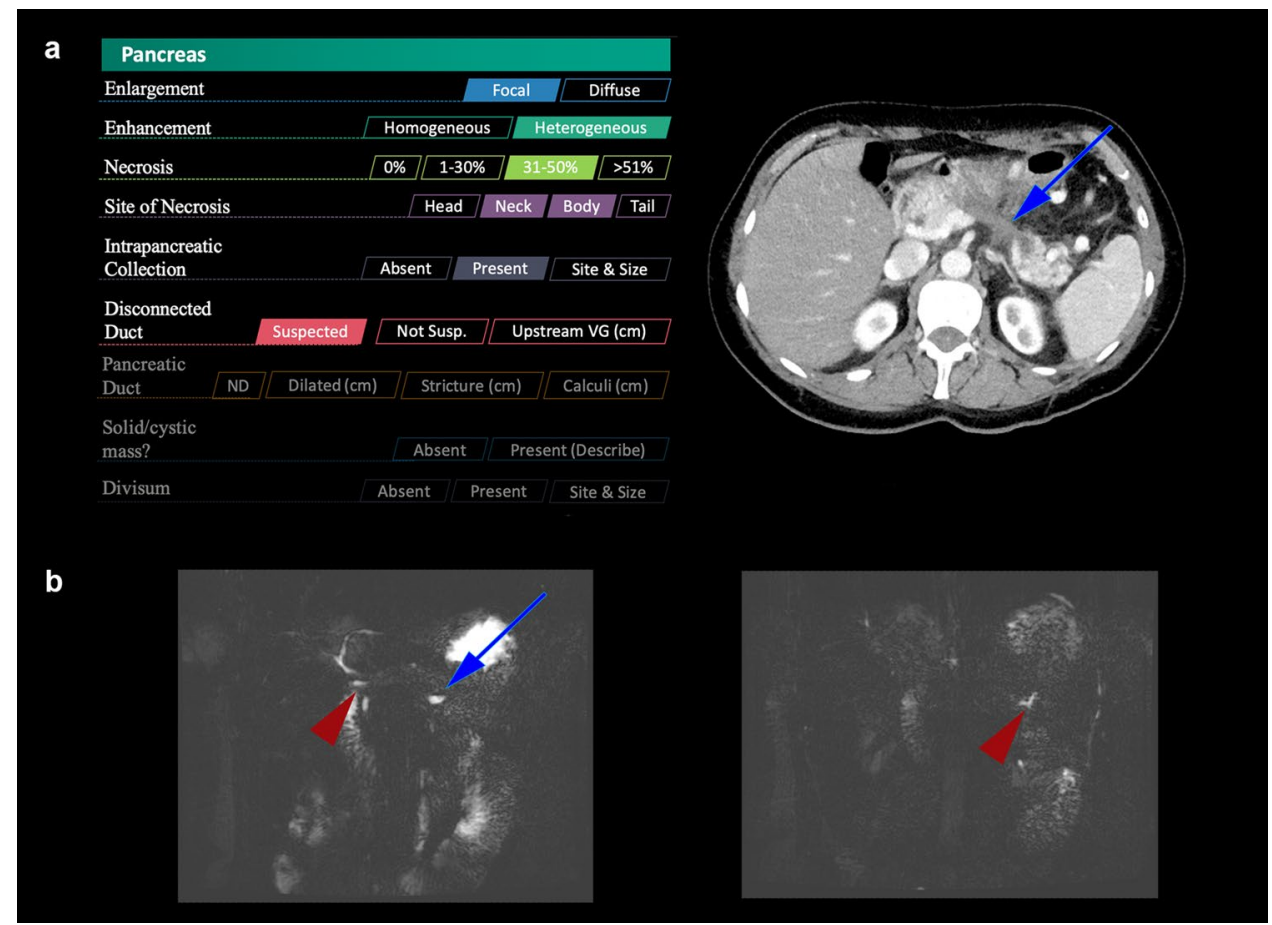


Fig. 6 a Proposed template and axial CECT image of an acute peripancreatic collection with non-encapsulated fluid within the transverse mesocolon (arrow) and left anterior pararenal space $\mathbf{b}$ proposed template and axial CECT image of a peripancreatic pseudocyst with mass effect (arrows) in a patient with an episode of acute pancreatitis more than 4 weeks prior to imaging

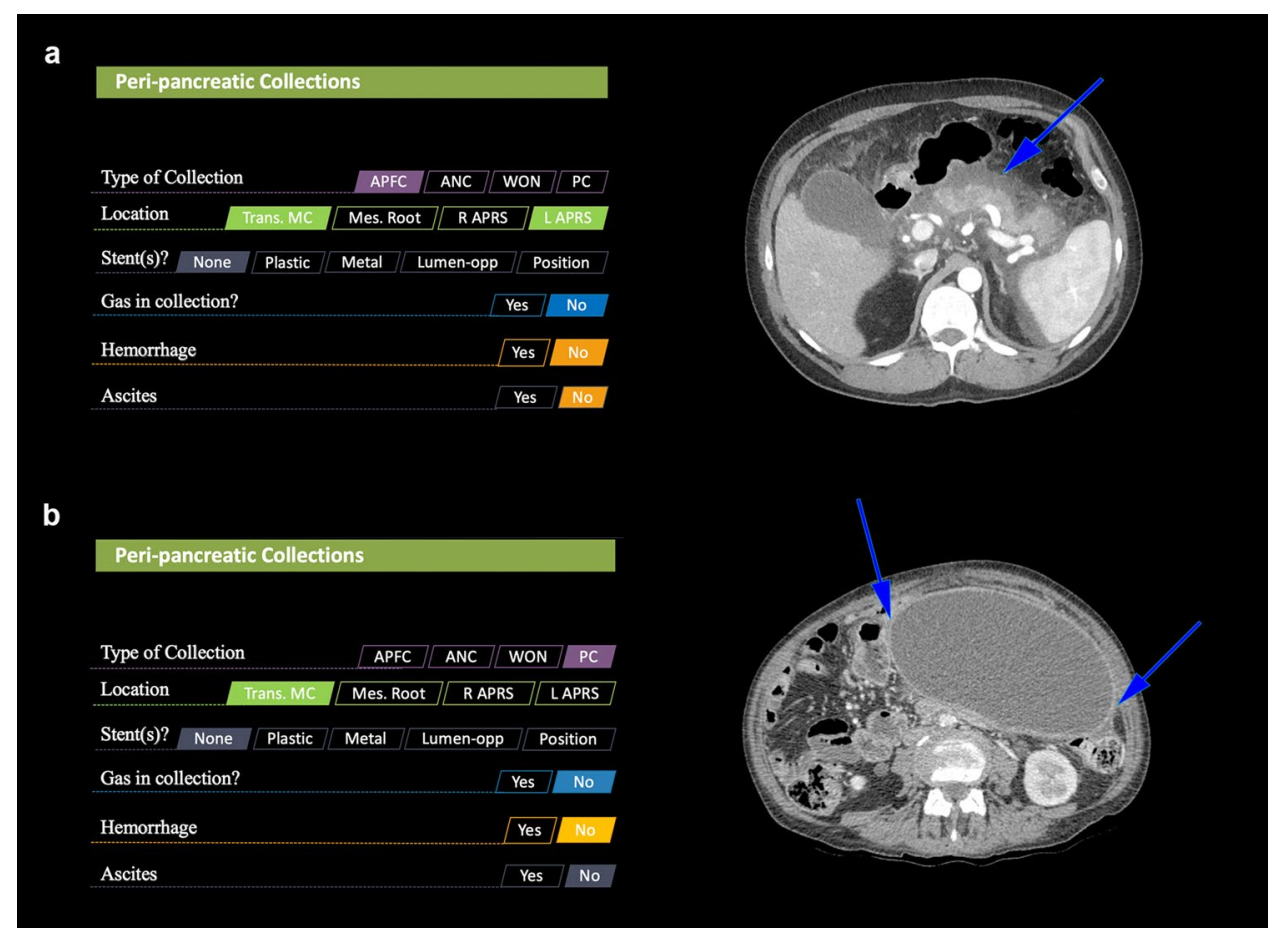

Fig. 7 a Proposed template and axial CECT showing necrotizing pancreatitis with adjacent heterogeneous encapsulated walled off necrosis with internal fat components (arrow) in the transverse mesocolon in a patient with an episode of acute pancreatitis more than 4 weeks prior to imaging and $\mathbf{b}$ Proposed template and axial CECT showing necrotizing pancreatitis with adjacent walled off necrosis with foci of gas (arrow) in the transverse mesocolon and left anterior pararenal space

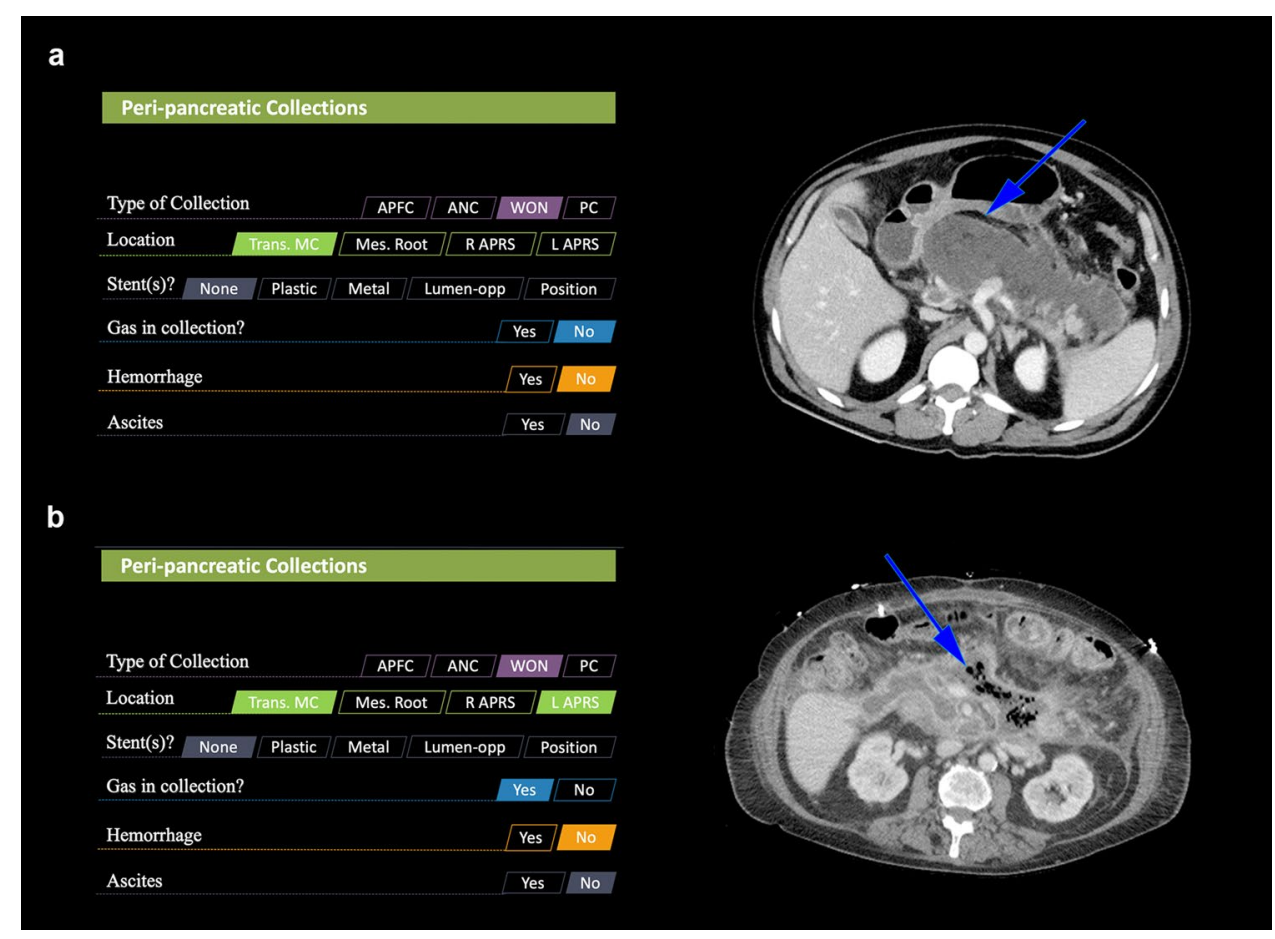

of the peripancreatic collection is crucial for gastroenterologists and interventional radiologists to plan the drainage route and feasibility. It may be subclassified as within transverse mesocolon, mesenteric root, and right or left anterior pararenal spaces. Presence of gas and hemorrhage within the collection are other important findings which predict higher morbidity and mortality and thus deserve attention in this template. The determination of an infectious process is important for diagnosis as there is a higher likelihood for treatment and intervention if it is present [8]. Thus it is critical to have appropriate communication with the patient's primary care team. Collections that demonstrate non-liquified material are more likely to be infected or secondary to fat necrosis. However, the best indicator for infection 
Fig. 8 a Proposed template and axial CECT showing a large peripancreatic pseudocyst with non-dependent gas (arrow) b Proposed template and axial unenhanced $\mathrm{CT}$ showing a heterogeneously attenuating pseudocyst with adherent blood products (arrow)
Fig. 9 a Proposed template and axial CECT showing extensive upper abdominal varices (arrow) secondary to splenic vein thrombosis due to infected necrosis b Proposed template and curved planar reformation of CT angiogram showing a splenic artery pseudoaneurysm (arrow) secondary to infected necrosis

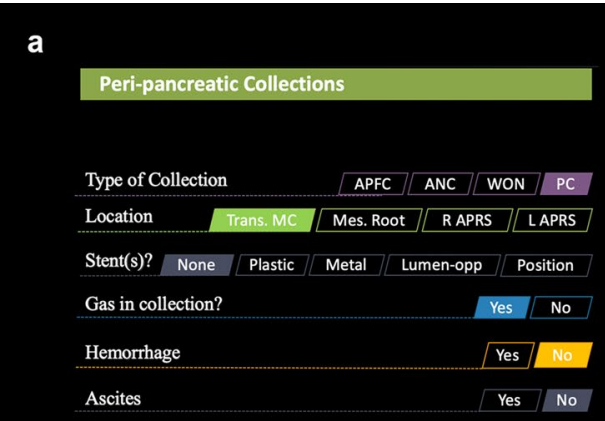

b

Peri-pancreatic Collections

\begin{tabular}{lll|l|l|} 
Type of Collection $\quad$ APFC & ANC & WON & PC \\
\hline
\end{tabular} \begin{tabular}{l|l|l|l|l|l|l|l|l}
\hline Location & Trans. MC Mes. Root R RPRS LAPRS \\
\hline
\end{tabular} \begin{tabular}{ll|l|l|l|l|l|l} 
Stent(s)? None Plastic Metal Lumen-opp Position \\
\hline
\end{tabular} Gas in collection? \begin{tabular}{|l|l|}
\hline Yes & No \\
\hline
\end{tabular}

Hemorrhage Yes No Ascites Yes No
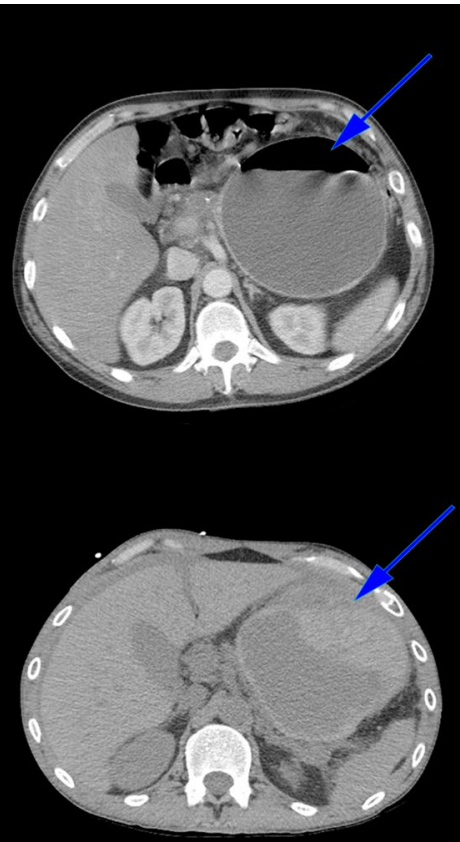

a

\section{Upper Abdominal Vasculature}

Thrombosis Splenic SMV PV

Perigastric varices

Yes No

Pseudoaneurysm?

Splenic Art

GDA

No

b

Upper Abdominal Vasculature

Thrombosis Splenic SMV PV

Perigatsric varices

Yes No

Pseudoaneurysm?

Splenic Art

GDA
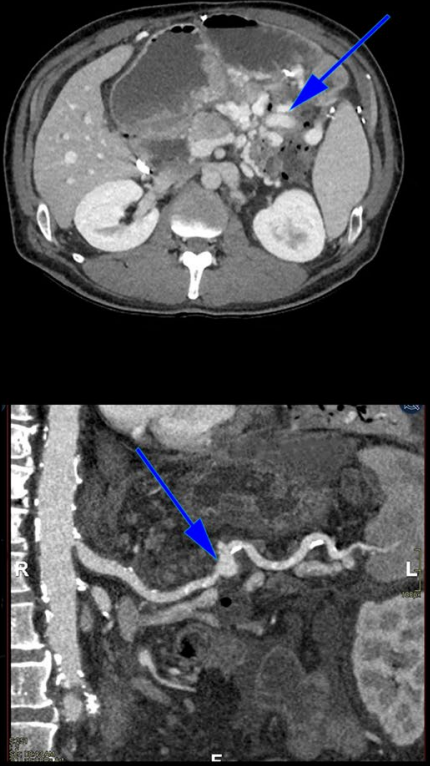
on CT imaging is the presence of gas in the fluid collections although often not seen [45] (Figs. 4, 8). Of note, it is important to thoroughly evaluate for gas caused by fistula, spontaneous drainage into the gastrointestinal tract and/or prior intervention to avoid false positive findings [29]. If there is no gas present in the collection and there remains a high suspicion, diagnostic proof may be obtained by performing fine need aspiration for Gram stain and culture of fluid [46]. Lastly, the presence of pancreatic stent and its positioning are included to aid clinicians in deciding further patient management.

A brief yet important category in this proposed template is focused on upper abdominal vasculature. The pancreas is surrounded by crucial mesenteric and splenic vasculature which are commonly involved in acute or resolving pancreatitis. The splenic, superior mesenteric and portal veins can be thrombosed based on the location of parenchymal inflammation and/or necrosis. Perigastric varices can be a subtle indicator of underlying splenic vein thrombosis in patients with severe inflammation or intrapancreatic fluid collections which could make direct vascular evaluation difficult (Fig. 9). Pseudoaneurysms occur from erosion of acute necrotic collections, walled off necrosis and pseudocysts into adjacent vasculature (Fig. 9). These take time to occur and therefore do not present in early disease but are associated with high mortality rates $(\sim 12 \%)$. Typical clinical presentations include hemorrhage within the gastrointestinal tract or within intraperitoneal spaces.

Last but not the least, the proposed template includes a section for adding free text in the midst of a very templated report. This will allow the radiologists to comment on ancillary findings such as gastric or left colonic wall thickening, duodenal narrowing and edema, presence of gallstones, biliary ductal dilation etc. This section also provides space to explain any of the above findings or complications in further detail. Comparisons to prior studies can also be described at length in this section.

\section{Conclusion}

Acute pancreatitis is a dynamic disease with various imaging presentations leading to important clinical management decisions. Imaging can aid in stratification of patients, particularly in identifying pancreatic and peripancreatic necrosis. In this paper, we tried to comprehensively discuss the historic and current classifications of acute pancreatitis and propose a new reporting template which fosters communication between different medical teams by use of appropriate terminology and structured radiology template. The standardized reporting template reduces ambiguity in radiologist's reports while allowing creation of a structured data repository for future research and teaching purposes.

The Revised Atlanta Classification has been adopted by various academic and private practice centers all over the world but still differences exist between the reports of a general radiologist and an expert when describing various components of acute pancreatitis. We aim to bridge those differences by offering a structured radiology report which provides a comprehensive step-by-step approach in reporting cases of acute pancreatitis and allows radiologists to add important information as free-text at the end of the template. Needless to say, specific larger studies are needed to validate improved outcomes in patients with acute pancreatitis for which standardized reporting template is used.

Acknowledgements The authors had control of the data and the information submitted for publication. Bhavik Patel receives research support from GE Healthcare and is on its speaker's bureau. Temel Tirkes is supported by National Cancer Institute and National Institute of Diabetes and Digestive and Kidney Diseases of the National Institutes of Health under Award Numbers 1R01DK116963 and U01DK108323 (Consortium for the Study of Chronic Pancreatitis, Diabetes, and Pancreatic Cancer). The content is solely the responsibility of the authors and does not necessarily represent the official views of the National Institutes of Health. All other authors are not employees of or consultants for industry and had control of inclusion of any data and information that might present a conflict of interest. There was no industry support specifically for this study.

\section{Compliance with ethical standards}

Ethical approval This study did not involve human and animal subjects, and therefore an Institutional Review Board approval and informed consent were not required.

\section{Appendix}

See Table 2. 
Table 2 Entire proposed pancreatitis template with all four broad categories of the reporting template-pancreas, peripancreatic fluid collections, upper abdominal vasculature \& other

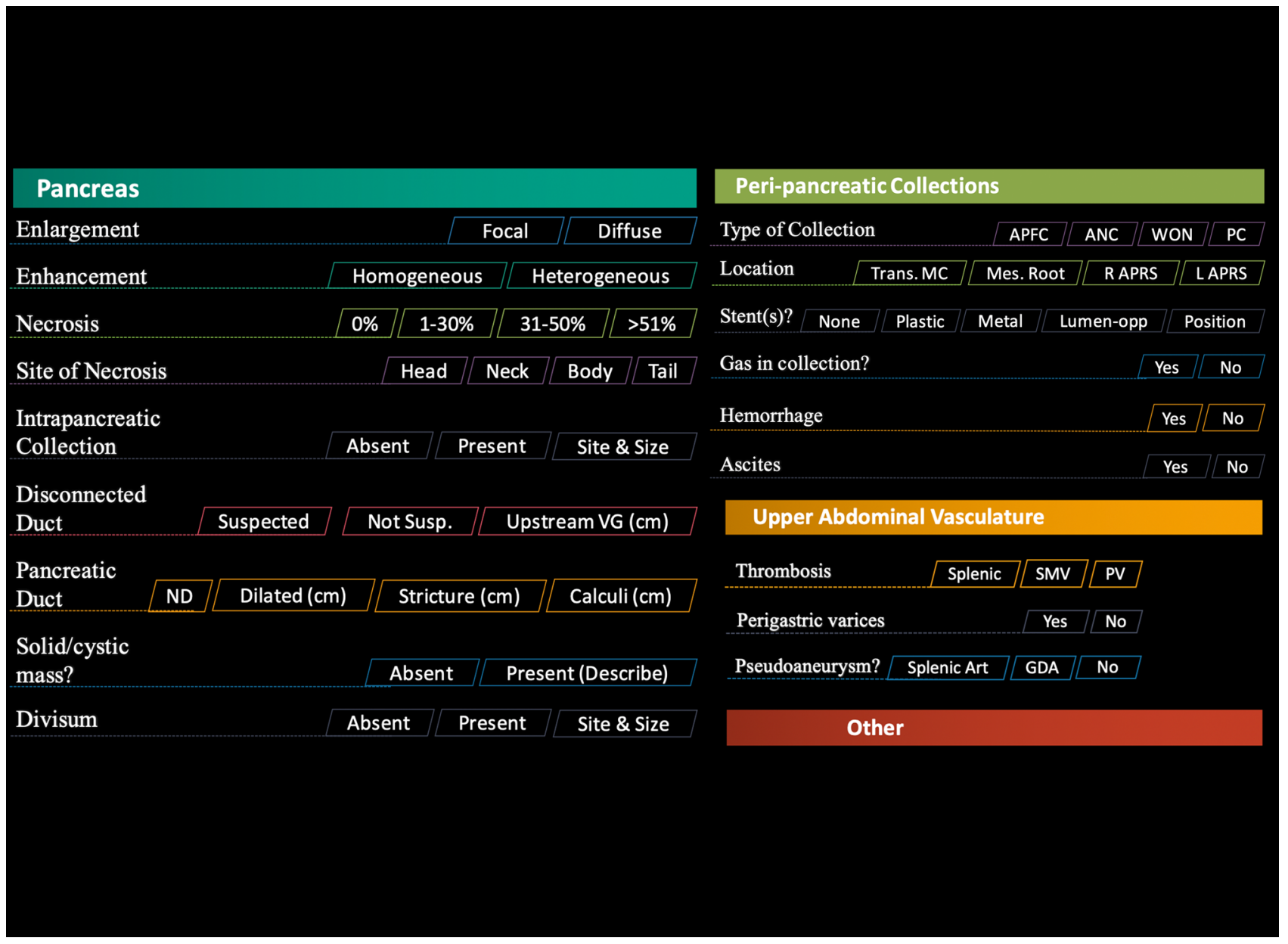

\section{References}

1. Bollen TL (2016) Acute pancreatitis: international classification and nomenclature. Clin Radiol 71:121-133. https://doi. org/10.1016/j.crad.2015.09.013

2. Murphy KP, O'Connor OJ, Maher MM (2014) Updated Imaging Nomenclature for Acute Pancreatitis. Am J Roentgenol 203:W464-W469. https://doi.org/10.2214/AJR.13.12222

3. Gapp J, Hall AG, Walters RW, et al (2019) Trends and Outcomes of Hospitalizations Related to Acute Pancreatitis: Epidemiology From 2001 to 2014 in the United States. Pancreas 48:548-554. https://doi.org/10.1097/MPA.0000000000001275

4. Balani AR, Grendell JH (2008) Drug-Induced Pancreatitis: Incidence, Management and Prevention. Drug Saf 31:823-837. https ://doi.org/10.2165/00002018-200831100-00002

5. Zhao K, Adam SZ, Keswani RN, et al (2015) Acute Pancreatitis: Revised Atlanta Classification and the Role of Cross-Sectional Imaging. Am J Roentgenol 205:W32-W41. https://doi. org/10.2214/AJR.14.14056

6. Zaheer A, Singh VK, Qureshi RO, Fishman EK (2013) The revised Atlanta classification for acute pancreatitis: updates in imaging terminology and guidelines. Abdom Imaging 38:125136. https://doi.org/10.1007/s00261-012-9908-0

7. Foster BR, Jensen KK, Bakis G, et al (2016) Revised Atlanta Classification for Acute Pancreatitis: A Pictorial Essay. RadioGraphics 36:675-687. https://doi.org/10.1148/rg.2016150097
8. Banks PA, Bollen TL, Dervenis C, et al (2013) Classification of acute pancreatitis - 2012: revision of the Atlanta classification and definitions by international consensus. Gut 62:102-111. https:// doi.org/10.1136/gutjnl-2012-302779

9. Garg SK, Sarvepalli S, Campbell JP, et al (2019) Incidence, Admission Rates, and Predictors, and Economic Burden of Adult Emergency Visits for Acute Pancreatitis: Data From the National Emergency Department Sample, 2006 to 2012. J Clin Gastroenterol 53:220-225. https://doi.org/10.1097/MCG.0000000000 001030

10. Khatua B, El-Kurdi B, Singh VP (2017) Obesity and pancreatitis: Curr Opin Gastroenterol 33:374-382. https://doi.org/10.1097/ MOG.0000000000000386

11. Peery AF, Crockett SD, Murphy CC, et al (2019) Burden and Cost of Gastrointestinal, Liver, and Pancreatic Diseases in the United States: Update 2018. Gastroenterology 156:254-272.e11. https:// doi.org/10.1053/j.gastro.2018.08.063

12. Bradley EL (1993) A Clinically Based Classification System for Acute Pancreatitis: Summary of the International Symposium on Acute Pancreatitis, Atlanta, Ga, September 11 Through 13, 1992. Arch Surg 128:586. https://doi.org/10.1001/archsurg.1993.01420 170122019

13. Besselink MGH, van Santvoort HC, Bollen TL, et al (2006) Describing Computed Tomography Findings in Acute Necrotizing Pancreatitis With the Atlanta Classification: An 
Interobserver Agreement Study. Pancreas 33:331-335. https:// doi.org/10.1097/01.mpa.0000240598.88193.8e

14. Bittner R, Block S, Büchler M, Beger HG (1987) Pancreatic abscess and infected pancreatic necrosis: Different local septic complications in acute pancreatitis. Dig Dis Sci 32:1082-1087. https://doi.org/10.1007/BF01300192

15. Colvin SD, Smith EN, Morgan DE, Porter KK (2019) Acute pancreatitis: an update on the revised Atlanta classification. Abdom Radiol. https://doi.org/10.1007/s00261-019-02214-w

16. Forsmark CE, Baillie J (2007) AGA Institute Technical Review on Acute Pancreatitis. Gastroenterology 132:2022-2044. https:// doi.org/10.1053/j.gastro.2007.03.065

17. Johnson CD (2004) Persistent organ failure during the first week as a marker of fatal outcome in acute pancreatitis. Gut 53:1340 1344. https://doi.org/10.1136/gut.2004.039883

18. Fleszler F, Braitman LE, Friedenberg F, et al (2003) Abdominal Computed Tomography Prolongs Length of Stay and Is Frequently Unnecessary in the Evaluation of Acute Pancreatitis. Am J Med Sci 325:251-255. https://doi.org/10.1097/00000441-20030 5000-00001

19. (2013) IAP/APA evidence-based guidelines for the management of acute pancreatitis. Pancreatology 13:e1-e15. https://doi. org/10.1016/j.pan.2013.07.063

20. Banks PA, Freeman ML, the Practice Parameters Committee of the American College of Gastroenterology (2006) Practice Guidelines in Acute Pancreatitis. Am J Gastroenterol 101:2379-2400. https://doi.org/10.1111/j.1572-0241.2006.00856.x

21. Zerem D, Zerema O, Zerem E (2017) Role of Clinical, Biochemi$\mathrm{cal}$, and Imaging Parameters in predicting the Severity of Acute Pancreatitis. Euroasian J Hepato-Gastroenterol 7:1-5. https://doi. org/10.5005/jp-journals-10018-1202

22. Spanier BWM, Nio Y, van der Hulst RWM, et al (2010) Practice and Yield of Early CT Scan in Acute Pancreatitis: A Dutch Observational Multicenter Study. Pancreatology 10:222-228. https:// doi.org/10.1159/000243731

23. Kamal A, Singh VK, Akshintala VS, et al (2015) CT and MRI assessment of symptomatic organized pancreatic fluid collections and pancreatic duct disruption: an interreader variability study using the revised Atlanta classification 2012. Abdom Imaging 40:1608-1616. https://doi.org/10.1007/s00261-014-0303-x

24. Koutroumpakis E, Slivka A, Furlan A, et al (2017) Management and outcomes of acute pancreatitis patients over the last decade: A US tertiary-center experience. Pancreatology 17:32-40. https ://doi.org/10.1016/j.pan.2016.10.011

25. Ocampo C, Zandalazini H, Kohan G, et al (2009) Computed Tomographic Prognostic Factors for Predicting Local Complications in Patients With Pancreatic Necrosis: Pancreas 38:137-142. https://doi.org/10.1097/MPA.0b013e31818de20a

26. Simchuk EJ, Traverso LW, Nukui Y, Kozarek RA (2000) Computed tomography severity index is a predictor of outcomes for severe pancreatitis. Am J Surg 179:352-355. https://doi. org/10.1016/S0002-9610(00)00375-5

27. Mikó A, Vigh É, Mátrai P, et al (2019) Computed Tomography Severity Index vs. Other Indices in the Prediction of Severity and Mortality in Acute Pancreatitis: A Predictive Accuracy Metaanalysis. Front Physiol 10:1002. https://doi.org/10.3389/fphys 2019.01002

28. Zerem E (2014) Treatment of severe acute pancreatitis and its complications. World J Gastroenterol 20:13879. https://doi. org/10.3748/wjg.v20.i38.13879

29. Thoeni RF (2012) The Revised Atlanta Classification of Acute Pancreatitis: Its Importance for the Radiologist and Its Effect on Treatment. Radiology 262:751-764. https://doi.org/10.1148/radio 1.11110947

30. Talukdar R, Bhattacharrya A, Rao B, et al (2014) Clinical utility of the Revised Atlanta Classification of acute pancreatitis in a prospective cohort: Have all loose ends been tied? Pancreatology 14:257-262. https://doi.org/10.1016/j.pan.2014.06.003

31. Sternby H, Verdonk RC, Aguilar G, et al (2016) Significant interobserver variation in the diagnosis of extrapancreatic necrosis and type of pancreatic collections in acute pancreatitis - An international multicenter evaluation of the revised Atlanta classification. Pancreatology 16:791-797. https://doi.org/10.1016/j. pan.2016.08.007

32. RSNA Radiology Reporting Initiative - Reporting Wiki. http://repor tingwiki.rsna.org/index.php/RSNA_Radiology_Reporting_Initiative \#RSNA.27s_Radiology_Report_Templates. Accessed 22 Oct 2019

33. Park J, Pillarisetty VG, Brennan MF, et al (2010) Electronic synoptic operative reporting: assessing the reliability and completeness of synoptic reports for pancreatic resection. J Am Coll Surg 211:308-315. https://doi.org/10.1016/j.jamcollsurg.2010.05.008

34. Schwartz LH, Panicek DM, Berk AR, et al (2011) Improving Communication of Diagnostic Radiology Findings through Structured Reporting. Radiology 260:174-181. https://doi.org/10.1148/ radiol.11101913

35. Brook OR, Brook A, Vollmer CM, et al (2015) Structured reporting of multiphasic CT for pancreatic cancer: potential effect on staging and surgical planning. Radiology 274:464-472. https:// doi.org/10.1148/radiol.14140206

36. Weiser TG, Haynes AB, Dziekan G, et al (2010) Effect of a 19-item surgical safety checklist during urgent operations in a global patient population. Ann Surg 251:976-980. https://doi. org/10.1097/SLA.0b013e3181d970e3

37. Levin DC (2012) Checklists: from the cockpit to the radiology department. J Am Coll Radiol JACR 9:388-390. https://doi. org/10.1016/j.jacr.2011.12.022

38. Kruskal JB, Reedy A, Pascal L, et al (2012) Quality initiatives: lean approach to improving performance and efficiency in a radiology department. Radiogr Rev Publ Radiol Soc N Am Inc 32:573-587. https://doi.org/10.1148/rg.322115128

39. Larson DB, Towbin AJ, Pryor RM, Donnelly LF (2013) Improving Consistency in Radiology Reporting through the Use of Department-wide Standardized Structured Reporting. Radiology 267:240-250. https://doi.org/10.1148/radiol.12121502

40. Sabo A, Goussous N, Sardana N, et al (2015) Necrotizing pancreatitis: a review of multidisciplinary management. JOP J Pancreas 16:125-135. https://doi.org/10.6092/1590-8577/2947

41. Sandrasegaran K, Tann M, Jennings SG, et al (2007) Disconnection of the pancreatic duct: an important but overlooked complication of severe acute pancreatitis. Radiogr Rev Publ Radiol Soc N Am Inc 27:1389-1400. https://doi.org/10.1148/rg.275065163

42. Chen Y, Jiang Y, Qian W, et al (2019) Endoscopic transpapillary drainage in disconnected pancreatic duct syndrome after acute pancreatitis and trauma: long-term outcomes in 31 patients. BMC Gastroenterol 19:54. https://doi.org/10.1186/s12876-019-0977-1

43. Tirkes T, Sandrasegaran K, Sanyal R, et al (2013) Secretin-enhanced MR Cholangiopancreatography: Spectrum of Findings. RadioGraphics 33:1889-1906. https://doi.org/10.1148/rg.337125014

44. Akisik MF, Sandrasegaran K, Aisen AA, et al (2006) Dynamic Secretin-enhanced MR Cholangiopancreatography. RadioGraphics 26:665-677. https://doi.org/10.1148/rg.263055077

45. Vege SS (2010) Peripancreatic collections in acute pancreatitis: Correlation between computerized tomography and operative findings. World J Gastroenterol 16:4291. https://doi.org/10.3748/ wjg.v16.i34.4291

46. Ashley SW, Perez A, Pierce EA, et al (2001) Necrotizing Pancreatitis: Contemporary Analysis of 99 Consecutive Cases. Ann Surg 234:572-580. https://doi.org/10.1097/00000658-20011 0000-00016

Publisher's Note Springer Nature remains neutral with regard to jurisdictional claims in published maps and institutional affiliations. 